\title{
THE FIRST AND SECOND-ORDER THEORY OF SHEARING AND COMPRESSION IN CASE OF THE BEAM FIXED AT ONE END AND SUPPORTED TO HELICAL SPRING AT THE OTHER END
}

\author{
Cornel Marin ${ }^{1}$ \\ ${ }^{1}$ VALAHIA University Of Târgoviște, FIMM, Aleea Sinaia Nr.13 Targoviste ROMANIA \\ marincor@yahoo.com
}

\begin{abstract}
The purpose of this paper is to present the results of two methods of calculating beams subjected simultaneously to bending and compression: no taking into account the influence of the beam displacements on momentum of compressive force (first order theory) and taking into account (second order theory). The relations obtained for one particular case in the MATHCAD program and we obtained the diagrams of the sectional shear and bending efforts, and the diagrams of deformation beam (displacements and slopes). These diagrams allow the comparative study of the results obtained by the two mentioned theory. We also simulate the position of the transverse force on the beam so that the maximum displacement is obtained, by the first theory and the second theory of calculus.
\end{abstract}

Keywords: Beam, Bending, Compression, Second-Order Theory.

\section{Introduction}

The stability calculus of elastic beams subjected to compressive and bending loads are solving using two methods: the first order theory - without taking into account the effect of compression force - and the second order theory - taking into account the effect of compression force [3], [7], [8].

In this chapter we consider the particular case, a simple straight beam with profiled standard section, having the section area

$A$ and axial moments of inertia $I_{y}$ know, which is fixed at one end and supported elastic at the other.

The beam is subject to bending and compression simultaneous loads by the transverse compressive force $Q$ and the axial force $P$ (smaller than buckling critical force $P_{c r}$ ).

We obtain the functions of bending moments, rotation and displacements for the curved fiber beams, according to the first order theory of calculus and also to the second order theory of calculus - i.e. taking into account the influence of compressive force.

These analytical expressions are introduced into MATHCAD professional program and we obtain the diagrams of bending moment $M_{1}(x)$, rotation $\varphi_{1}(x)$ and displacements $w_{1}(x)$ for first order theory.

Also, we obtain the diagrams of bending moment $M_{2}(x)$, rotation $\varphi_{2}(x)$ and the displacements $w_{2}(x)$ for second order theory.

\section{The First Order Theory for Calculus of Beam Subjected to Compressive and Bending Loads}

In Figure 1 it consider a particular case of straight beam of length $L$, having the section area $A$ and axial moments of inertia $I_{y}$ known. For this case, we wrote the functions of bending moments, of rotation and displacements for the beams, according to the firstorder theory - i.e. without taking into account the influence of compressive force on bending moments.

The beam is subjected to bending and compression loads by the external moment $N$, the transverse force $F$, the uniformly distributed force $q$ and one axial compression force $P$ - whose effect on bending moments can be neglected [2], [3].

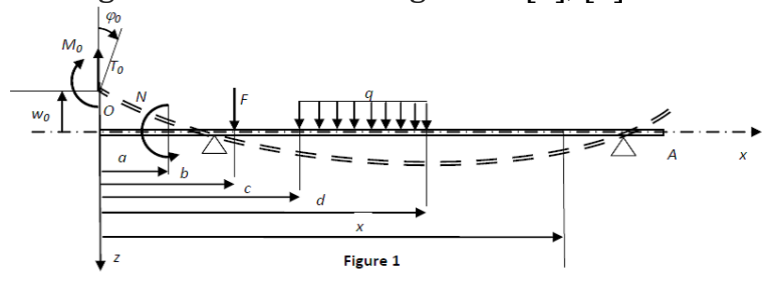

Figure 1

In Figure 1 we note:

- $w_{0}, \varphi_{0}$ - the displacement and rotating of the left end of the beam;

- $T_{0}, M_{0}$ - the corresponding shear forces and bending moment for the left end of the beam. 
We obtain the displacement $w_{1}(x)$ and rotating $\varphi_{1}(x)$ functions as solution of the differential equation of curved fiber of beam [1], [4], [5], [6]:

$\frac{d^{2} w}{d x^{2}}=-\frac{M_{i y}(x)}{E \cdot I_{y}}$
Where the bending moment $M_{i y}(x)$ function is written using step function $\Phi(x-a)$ of MATHCAD program, as particular relationship for this case:

$$
\begin{aligned}
M_{i y}(x)= & M_{0} \cdot \Phi(x)+N \cdot \Phi(x-a)+T_{0} \cdot x \cdot \Phi(x)-F \cdot(x-b) \cdot \Phi(x-b)- \\
& -\frac{q_{0}}{2} \cdot \Phi(x-c) \cdot(x-c)^{2}+\frac{q}{2} \cdot \Phi(x-d) \cdot(x-d)^{2}
\end{aligned}
$$

By integrating the differential equation (1) we obtain the rotation function $\varphi 1(x)$ of the section beam using step function $\Phi(x-a)$; for this purpose we

$$
\begin{aligned}
& \frac{d w_{l}(x)}{d x}=\tan \left[\varphi_{1}(x)\right] \cong \varphi_{1}(x) \\
& \varphi_{1}(x)=\frac{d w_{l}(x)}{d x}=\varphi_{0}-\frac{1}{E I_{y}}\left[M_{0} \cdot x \cdot \Phi(x)+N \cdot(x-a) \cdot \Phi(x-a)+\frac{T_{0}}{2} \cdot x^{2} \cdot \Phi(x)-\right. \\
& \left.\quad-\frac{F}{2}(x-b)^{2} \cdot \Phi(x-b)-\frac{q_{0}}{6} \cdot(x-c)^{3} \cdot \Phi(x-c)+\frac{q}{6} \cdot(x-d)^{3} \cdot \Phi(x-d)\right]
\end{aligned}
$$

By integrating the differential relation (3) we obtain the displacement function $\mathrm{w} 1(\mathrm{x})$ of the curved beam:

$$
\begin{aligned}
w l(x)= & w_{0}+\varphi_{0} \cdot x-\frac{1}{E I_{y}}\left[\frac{M_{0}}{2} \cdot x^{2} \cdot \Phi(x)+\frac{N}{2} \cdot(x-a)^{2} \cdot \Phi(x-a)+\frac{T_{0}}{6} \cdot x^{3} \cdot \Phi(x)-\right. \\
& \left.-\frac{F}{6}(x-b)^{3} \cdot \Phi(x-b)-\frac{q_{0}}{24} \cdot(x-c)^{4} \cdot \Phi(x-c)+\frac{q}{24} \cdot(x-d)^{4} \cdot \Phi(x-d)\right] ;
\end{aligned}
$$

The expressions (2), (3) and (4) vas introduced into the MATHCAD professional program to draw the bending, rotation and displacement diagrams.

\section{The Second Order Theory for Calculus of Beam Subjected to Compressive and Bending Loads}

In Figure 2 it consider a same particular case of straight beam of length $\mathrm{L}$, having the section area $A$ and axial moments of inertia $I_{y}$ known.

For this particular case, we wrote the functions of bending moments, of rotation and displacements for the curved fibre beams, according to the secondorder theory- i.e. taking into account the influence of compressive force on bending moments.

The beam is subjected to bending and compression loads by the external moment $N$, the transverse force $F$, the uniformly distributed force $q$ and one axial compression force $\mathrm{P}$ - whose effect on bending moments cannot be neglected [3], [7], [8].

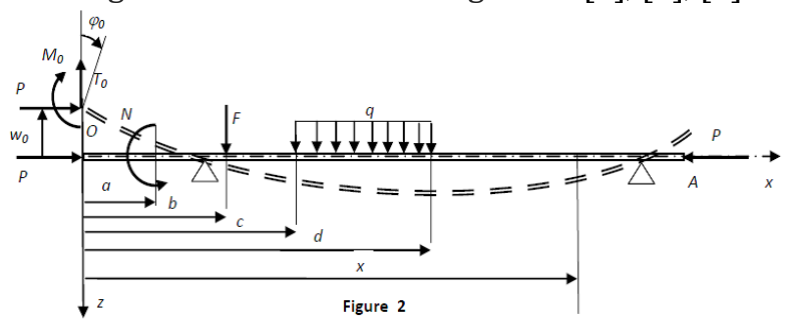

Figure 2
For this particular case we note also:

- $w 0, \varphi_{0}$ are the displacement and rotating of the left end of the beam;

- $T_{0}, M_{0}$ - the corresponding forces and moment of the left end of the beam.

We obtain the displacement $w_{2}(x)$ and rotating $\varphi_{2}(x)$ functions as solution of the differential equation of curved fiber of beam [1], [3]:

$\frac{d^{2} M_{i}}{d x^{2}}=-q+P \cdot \frac{d^{2} w}{d x^{2}}$

If we derivates twice the differential equation of curved fiber (1) and taking into account the relationship (5) we obtained the differential equation:

$$
\frac{d^{4} w}{d x^{4}}+\frac{P}{E \cdot I_{y}} \cdot \frac{d^{2} w}{d x^{2}}=q
$$

The solution of the differential equation (6) is written as follow:

$$
\begin{aligned}
& w 2(x)=C_{1} \sin (k x)+C_{2} \cos (k x)+C_{3} x+C_{4}+\bar{w} \\
& \varphi 2(x)=k C_{1} \cos (k x)-k C_{2} \sin (k x)+C_{3}+\frac{d w}{d x}
\end{aligned}
$$

If we derivates twice the differential equation (7) we obtained the bending moment function $M_{i}(x)$ respectively the shear functions $T(x)$ : 


$$
\begin{aligned}
& M 2(x)=-E I_{y}\left(-k^{2} C_{1} \sin (k x)-k^{2} C_{2} \cos k x+\frac{d^{2} \bar{w}}{d x^{2}}\right) \\
& T 2(x)=E I_{y}\left(k^{3} C_{1} \cos (k x)-k^{3} C_{2} \sin k x-\frac{d^{3} \bar{w}}{d x^{3}}\right)
\end{aligned}
$$

where: $k$ is one parameter depending of the compressive force $P$ :

$$
k^{2}=\frac{P}{E \cdot I_{y}}
$$

- $C_{1}, C_{2}, C_{3}, C_{4}$ are the constants determined from the boundary conditions of curved beam (displacements and rotations of the beam ends); $\bar{w}(x), \quad \bar{\varphi}(x)=\frac{d \bar{w}}{d x}$ are the particular solutions suitable for external loads $N, F, q$.

This particular solution for external loads $\mathrm{N}, \mathrm{F}$ and $\mathrm{q}$ is written [1], [3], [7], [8]:

$$
\begin{aligned}
& \bar{w}_{N}(x)=-\frac{N}{P}(1-\cos (k \cdot(x-a))) \quad \text { for } x \geq a \\
& \bar{w}_{F}(x)=\frac{F}{k P}(k \cdot(x-b)-\sin (k \cdot(x-b))) \quad \text { for } x \geq b \\
& \bar{w}_{q}(x)=\frac{q}{k P} \int_{c}^{d}(k \cdot(x-t)-\sin (k \cdot(x-t))) d t \quad \text { for } x \in(c, d)
\end{aligned}
$$

If the origin parameter values: $w_{0}, \varphi_{0}, M_{0}$ and $T_{0}$ are know, it can written the boundary conditions take into account the relationship (7) and (8):

$$
\begin{aligned}
& w_{2}(O)=w_{0}=C_{2}+C_{4} \\
& \varphi_{2}(x)=\varphi_{0}=k C_{1}+C_{3} \\
& M_{2}(O)=M_{0}=E \cdot I_{y}\left(k^{2} \cdot C_{2}\right) \\
& T_{2}(O)=T_{0}+P \cdot \varphi_{0}=E \cdot I_{y}\left(k^{3} \cdot C_{1}\right)
\end{aligned}
$$

The expressions of constants $C_{1}, C_{2}, C_{3}, C_{4}$ are obtained as followed:

$$
C_{1}=\frac{T_{0}}{k P}+\frac{\varphi_{0}}{k} ; \quad C_{2}=\frac{M_{0}}{E I_{y} \cdot k^{2}}=\frac{M_{0}}{P} ; \quad C_{3}=-\frac{T_{0}}{P} ; \quad C_{4}=w_{0}-\frac{M_{0}}{P}
$$

Substituting expressions functions (12) into relations (7) and (8) we obtained the relationship of displacement $w_{2}(x)$, rotating $\varphi_{2}(x)$, bending moment $M_{2}(x)$ and shear functions $T_{2}(x)$ functions:

$$
\begin{aligned}
& w_{2}(x)=w_{O}+\frac{\varphi_{0}}{k} \sin (k \cdot x)-\frac{M_{O}}{P}(1-\cos (k \cdot x))-\frac{T_{O}}{k \cdot P}(k \cdot x-\sin (k \cdot x))+\bar{w} \\
& \varphi_{2}(x)=\varphi_{0} \cdot \cos (k \cdot x)-\frac{M_{O}}{P} k \cdot \sin (k \cdot x)-\frac{T_{O}}{P}(1-\cos (k \cdot x))+\frac{d \bar{w}}{d x} \\
& M_{2}(x)=E I_{y}\left(\varphi_{O} \cdot k \cdot \sin (k \cdot x)+\frac{M_{O}}{P} k^{2} \cdot \cos (k \cdot x)+\frac{T_{O}}{P} k \cdot \sin (k \cdot x)-\frac{d^{2} \bar{w}}{d x^{2}}\right) \\
& T_{2}(x)=E I_{y}\left(\varphi_{O} \cdot k^{2} \cdot \cos (k \cdot x)-\frac{M_{O}}{P} k^{3} \cdot \sin (k \cdot x)+\frac{T_{O}}{P} k^{2} \cdot \cos (k \cdot x)-\frac{d^{3} \bar{w}}{d x^{3}}\right)
\end{aligned}
$$

\section{Particular Case Study of First and Second Order Theory of Calculus}

For particular case study we consider a straight beam from Figure 3 with length $L=5 \mathrm{~m}$, profiled standard section 118 (STAS 565-80) having section area $A=27.9 \mathrm{~cm}^{2}$ and axial moments of inertia $I_{y}=$ $81.3 \mathrm{~cm}^{4}\left(I_{z}=145 \mathrm{~cm}^{4}\right)$. The beam is fixed at one end and supported to helical spring at the other end. The beam is subject to bending and compression loads by one transverse force $Q=2,5 \mathrm{kN}$ applied to embedding distance $\alpha L(\alpha=0,5)$ and one axial force $P=0,25 \cdot P_{c r}$ ( $P_{c r}$ is the buckling critical force), at the other end.

The material beam has the following mechanical characteristics: longitudinal elastic modulus $E=$ $2,1 \cdot 10^{5} \mathrm{MPa}$; yield stress $\sigma_{c}=150 \mathrm{MPa}$, coefficient of thinness to buckling $\lambda_{0}=105$.
Cylindrical helical spring has the following characteristics: $d=18 \mathrm{~mm}, D=100 \mathrm{~mm}$, elastic shear modulus $G=85 \cdot 10^{3} \mathrm{MPa}, n=9$ rings.

The study was done for the following cases:

CASE 1: For first-order theory of calculus we calculate the reaction moment/forces $M_{0}, T_{0}, V_{A}$ and drawing the diagrams of moments, displacements and rotations, with (2), (3), (4) relationship and MATHCAD program facilities.

CASE 2: For second-order theory of calculus we calculate the reaction moment/forces $M_{0}, T_{0}, V_{A}$ and drawing the diagrams of moments, displacements and rotations, with (13) relationship and MATHCAD program facilities.

For first-order theory and second-order theory of calculus we drawing the comparative diagrams of bending moment, displacements and rotations. 


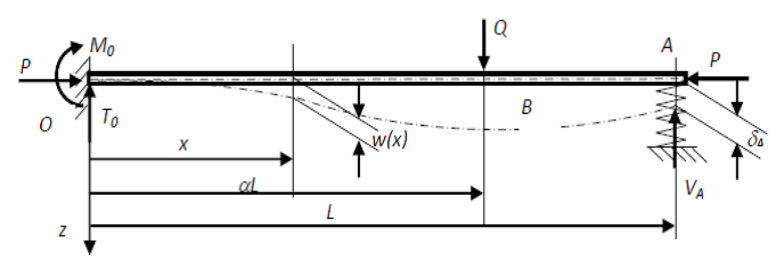

Figure 3

\section{CASE 1: The first-order theory of calculus}

The unknown reaction moment/forces $M_{0}, T_{0}, V_{A}$ are obtained from equilibrium equations of Mechanics, and the condition of proportionality of reaction $V_{A}$ with displacement $w_{A}[11]$, [12] (Figure 3):

$$
\begin{aligned}
& w_{A}=\delta_{A} \cdot V_{A} ; \quad \delta_{A}=\frac{8 \cdot D^{3} \cdot n}{G \cdot d^{4}} \\
& M 1(x)=M_{0} \cdot \Phi(x)+T_{0} \cdot x \cdot \Phi(x)-Q \cdot(x-\alpha L) \cdot \Phi(x-\alpha L)+V_{A} \cdot(x-L) \cdot \Phi(x-L)
\end{aligned}
$$

The equilibrium equations of Mechanics are written:

$$
\begin{array}{lll}
\sum F_{z}=0 & \Rightarrow & T_{0}+V_{A}-Q=0 \\
\sum M_{y O}=0 & \Rightarrow & M_{0}+Q \cdot \alpha L-V_{A} \cdot L=0
\end{array}
$$

The condition of proportionality (14) lead to expression of reaction $T_{0}$ :

$$
T_{0}=\frac{1-\alpha-\frac{(1-\alpha)^{3}}{3}+\frac{2 \cdot E \cdot I_{y}}{L^{3}} \cdot \delta_{A}}{\frac{2}{3}+\frac{2 \cdot E \cdot I_{y}}{L^{3}} \cdot \delta_{A}} \cdot Q
$$

The bending moment $M_{1}(x)$ function is written using step function $\Phi(x-a)$ of MATHCAD program, for this case as particular relationship:

The relationship (3) and (4) of rotation function $\varphi_{1}(x)$ and displacements function $w_{1}(x)$ are becoming:

$$
\begin{aligned}
& \varphi l(x)=-\frac{1}{E I_{y}}\left[M_{O} \cdot x \cdot \Phi(x)+T_{O} \cdot \frac{x^{2}}{2} \cdot \Phi(x)-Q \frac{(x-\alpha L)^{2} \cdot}{2} \Phi(x-\alpha L)+V_{A} \frac{(x-L)^{2}}{2} \cdot \Phi(x-L)\right] \\
& w l(x)=-\frac{1}{E I_{y}}\left[M_{O} \cdot \frac{x^{2}}{2} \cdot \Phi(x)+T_{0} \cdot \frac{x^{3}}{6} \cdot \Phi(x)-Q \frac{(x-\alpha L)^{3}}{6} \Phi(x-\alpha L)+V_{A} \frac{(x-L)^{3}}{6} \cdot \Phi(x-L)\right]
\end{aligned}
$$

The diagrams of moments, displacements and rotations for this particular case are obtained with relationship (17), (18) and MATHCAD program facilities; they are drawing in Figure 4.

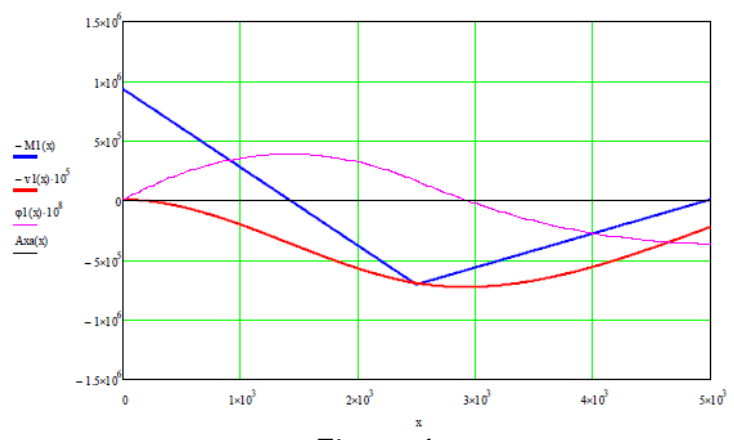

Figure 4

We can seen from the Figure 4 that the maximum bending effort is obtained for $x=\alpha L$ and the maximum displacement is obtained from condition:

$$
\frac{d \varphi l(x)}{d x}=0
$$

Using MATHCAD program facilities (root function) we obtain:

$$
\begin{aligned}
& M_{1}(\alpha L)=7.6466 \times 10^{5} \mathrm{Nmm} \\
& \operatorname{root}\left(\varphi_{1}(x), x, 2000,4000\right)=2.8631 \times 10^{3} \mathrm{~mm} \\
& w_{l_{\text {max }}}=7.446 \mathrm{~mm}
\end{aligned}
$$

\section{CASE 2: The second-order theory of calculus}

The reaction moment/forces $M_{0}, T_{0}, V_{A}$ are obtained from equilibrium equations of Mechanics (15), and boundary condition of displacement [1], [3]:

$$
w(L)=V_{A} \cdot \delta_{A}
$$$$
M_{i y}(L)=0
$$

The relationships (13) of displacements $w_{2}(x)$, rotation $\varphi_{2}(x)$, and bending moment $M_{2}(x)$, are writing for this particular case as follow:

$$
\begin{aligned}
& w_{2}(x)=-\frac{T_{0}}{k \cdot P}(k \cdot x-\sin (k \cdot x))-\frac{M_{0}}{P}(1-\cos (k \cdot x))+\frac{Q}{k \cdot P}(k(x-\alpha L)-\sin (k \cdot(x-\alpha L))) \cdot \Phi(x-\alpha L) \\
& \varphi_{2}(x)=-\frac{T_{0}}{P}(1-\cos (k \cdot x))-\frac{M_{0}}{P} \cdot k \cdot \sin (k \cdot x)+\frac{Q}{P}(1-\cos (k \cdot(x-\alpha L))) \cdot \Phi(x-\alpha L) \\
& M_{2}(x)=\frac{T_{0}}{k} \cdot \sin (k \cdot x)+M_{0} \cdot \cos (k \cdot x)+\frac{V_{B}}{k} \cdot \sin (k \cdot(x-\alpha L)) \cdot \Phi(x-\alpha L)-\frac{Q}{k} \cdot \cos (k \cdot(x-\alpha L)) \cdot \Phi(x-\alpha L)
\end{aligned}
$$


Taking into account the relationships is achieved the expression of $T_{0}, M_{0}, V_{A}$

$$
\begin{aligned}
& T_{0}=-Q \frac{(1-\cos (k L)) \cdot \sin (k L \cdot(1-\alpha))-\cos (k L) \cdot\left((1-\alpha) k L-\sin ((1-\alpha) k L)-\left(\frac{k L}{L}\right)^{3} E \cdot I_{y} \cdot \delta_{A}\right)}{(1-\cos (\alpha \cdot k L)) \cdot \sin (k L)-\cos (k L) \cdot\left(k L-\sin (k L)-\left(\frac{k L}{L}\right)^{3} E \cdot I_{y} \cdot \delta_{A}\right)} \\
& M_{0}=-Q \cdot L \frac{\sin (k L) \cdot\left((1-\alpha) k L-\sin ((1-\alpha) k L)-\left(\frac{k L}{L}\right)^{3} E \cdot I_{y} \cdot \delta_{A}\right)-\sin ((1-\alpha) k L) \cdot\left(k L-\sin (k L)-\left(\frac{k L}{L}\right)^{3} E \cdot I_{y} \cdot \delta_{A}\right)}{(1-\cos (\alpha \cdot k L)) \cdot \sin (k L)-\cos (k L) \cdot\left(k L-\sin (k L)-\left(\frac{k L}{L}\right)^{3} E \cdot I_{y} \cdot \delta_{A}\right)} \\
& V_{A}=Q-T_{0}
\end{aligned}
$$

The diagrams of moments, displacements and rotations for this particular case (the compressive force $P=0,25 \cdot P_{c r}$ ) are obtained with relationship (21) and MATHCAD program facilities; they are drawing in Figure 5.
We can seen from the Figure 5 that the maximum bending effort is obtained for

$x=\alpha L$ and the maximum displacement is obtained from condition: $\frac{d \varphi l(x)}{d x}=0$

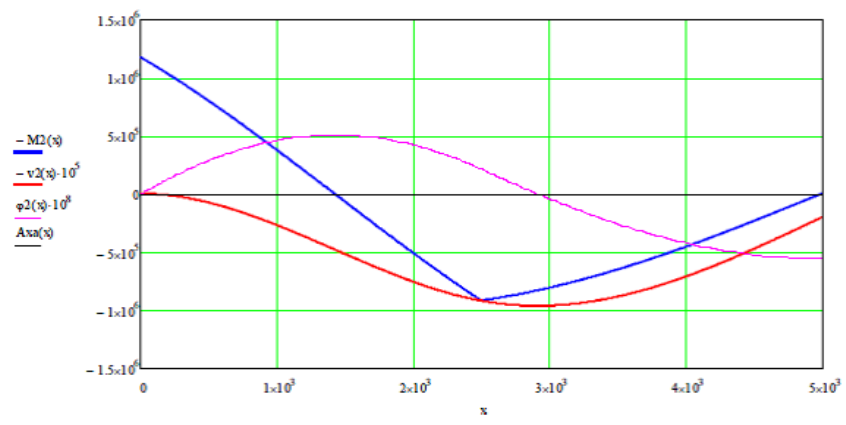

Figure 5

$$
\begin{aligned}
& M_{2}(\alpha L)=9.9185 \times 10^{5} \mathrm{Nmm} ; \operatorname{root}\left(\varphi_{2}(x), x, 2000,4000\right)=2.8776 \times 10^{3} \mathrm{~mm} \\
& w_{2 \max }=10.0195 \mathrm{~mm}
\end{aligned}
$$

The critical buckling forces $P_{c r}$ are obtained for this case from denominator zero condition of relationship (22) of reaction $T_{0}, M_{0}$. We note the denominator as function $F(k L)$ :

$$
F(k L)=(1-\cos (\alpha \cdot k L)) \cdot \sin (k L)-\cos (k L) \cdot\left(k L-\sin (k L)-\left(\frac{k L}{L}\right)^{3} E \cdot I_{y} \cdot \delta_{A}\right)=O
$$

The first solution is obtained with root function of MATHCAD program facilities:

$$
\operatorname{root}\left[\cos (\mathrm{kL}) \cdot\left[\mathrm{kL}-\mathrm{sin}(\mathrm{kL})-(\mathrm{kL})^{3} \cdot \frac{\mathrm{E} \cdot \mathrm{Iy}}{\mathrm{L}^{3}} \cdot \delta \mathrm{A}\right]-(1-\cos (\mathrm{kL})) \cdot \sin (\mathrm{kL}), \mathrm{kL}, 4,6\right]=4.4561
$$

We obtain in MATHCAD the numerical value of buckling forces fot this case:

$$
P_{c r}=\frac{(k L)^{2}}{L^{2}} \cdot E \cdot I_{y} \quad \Rightarrow P_{c r}=1.3561 \times 10^{5} \mathrm{~N}
$$

The value of axial force used in calculus is:

$$
P=0,25 \cdot P_{c r}=33,9025 \times 10^{3} \mathrm{~N}
$$

In order to represent the comparative diagrams of bending moment, displacements and rotations obtained with first-order theory (Figure 4) and second-order theory of calculus (Figure 5) are drawing with MATHCAD program facilities:

- The comparative diagrams of bending moment in Figure 6.

- The comparative diagrams of displacements in Figure 7.

- The comparative diagrams of rotations in Figure 8. 
The First and Second-Order Theory of Shearing and Compression in Case of the Beam Fixed at One End and Supported to Helical Spring at the Other End

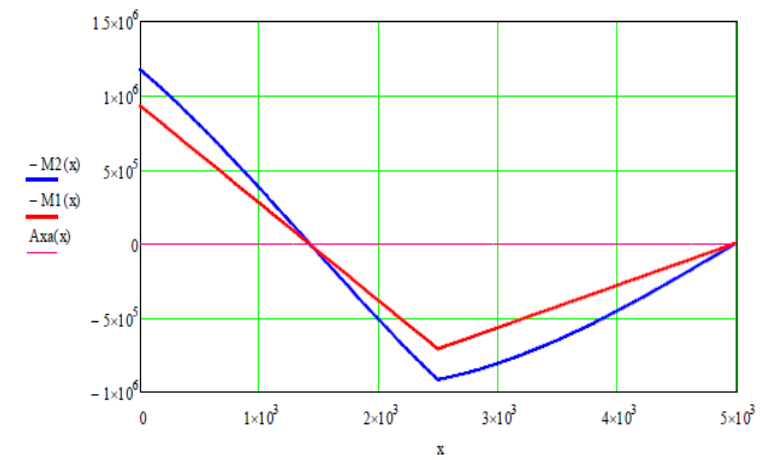

Figure 6: The comparative bending moment diagrams

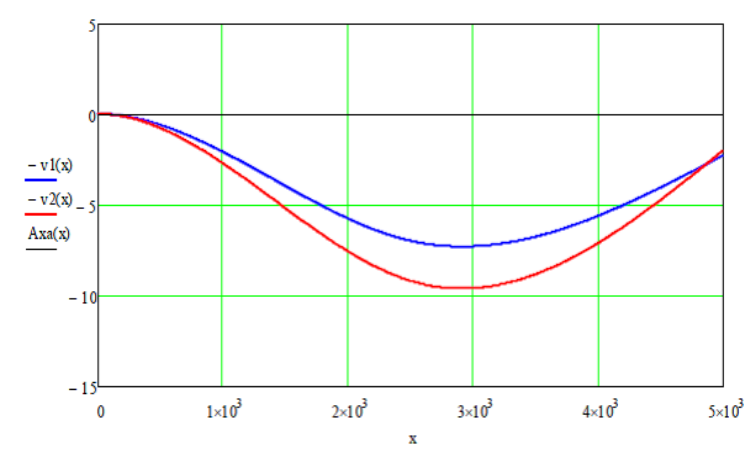

Figure 7: The comparative displacement diagrams

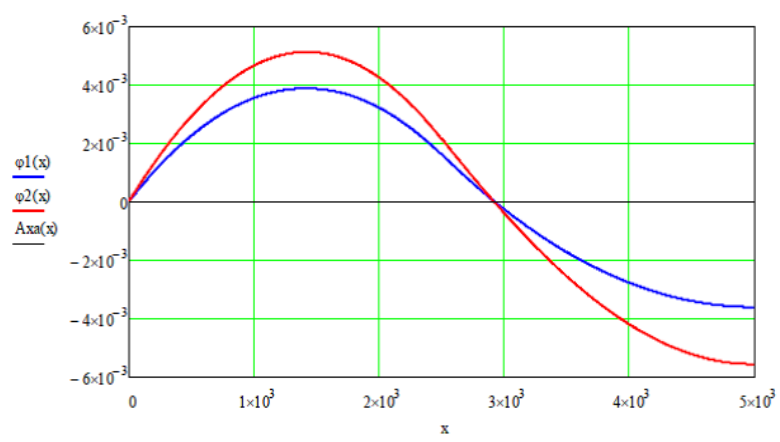

Figure 8: The comparative rotations diagrams

The numerical results obtained for the particular case presented in this section let to conclusion that the second-order theory is an accurate method and the diagrams obtained show that increasing compressive force lead to increasing values of moments, rotations and displacements compared to those obtained through the first-order theory. Figure 7 and Figure 8 show also that the position of section corresponding to maximum bending moment (the middle beam) is not located at same distance with the section corresponding to the maximum displacement.

Also, can be observed small difference for the position of section corresponding to the maximum displacement for first order theory $\left(x_{1 \max }=2,8631 \mathrm{~mm}\right)$ and second ordertheory $\left(x_{2 \max }=2,8776 \mathrm{~mm}\right)$ we obtained large differences between the displacements value $\left(v_{1 \max }=7,446 \mathrm{~mm}, v_{2 \max }=10,0195\right.$ $m m$ ).

\section{Simulation of Maximum Displacement Variation with Distance $\alpha L$ of External Force $Q$}

Figures $9 \quad \ldots 13$ shows the variation of bending moment, displacement and rotation corresponding to five different parameter values $\alpha=0,1 ; \alpha=0,3$; $\alpha=0,5 ; \alpha=0,7 ; \alpha=0,9$. So we found the position of the transverse force on the beam $Q$ so that is obtained the maximum displacement, both using the firstorder theory and the second-order theory of calculus.

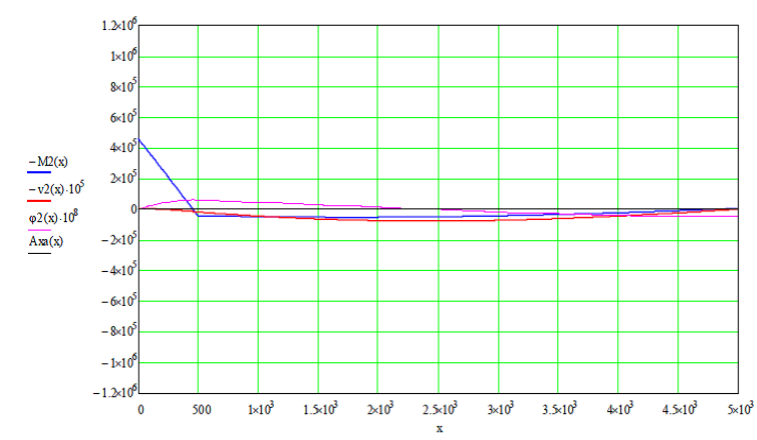

Figure 9: Variation of bending moment, displacements and rotations for $\alpha=0,1 x_{\max }=2435,9 \mathrm{~mm} ; V_{2 \max }=0,8165$ $\mathrm{mm}$.

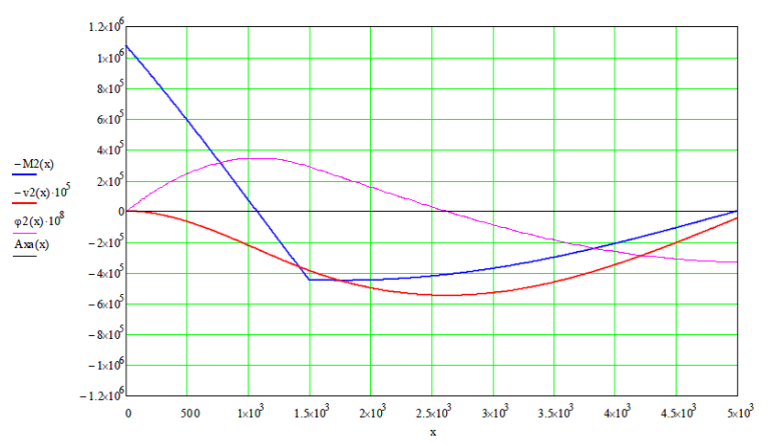

Figure 10: Variation of bending moment, displacements and rotations for $\alpha=0,3 x_{\max }=2625,1$ $\mathrm{mm} ; V_{2 \max }=5,4856 \mathrm{~mm}$.

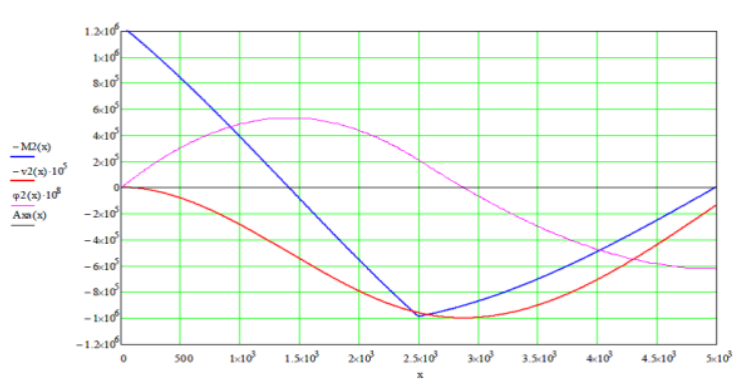

Figure 11: Variation of bending moment, displacements and rotations for $\alpha=0,5 x_{\max }=2877,6$ $\mathrm{mm} ; V_{2 \max }=10,0195 \mathrm{~mm}$. 


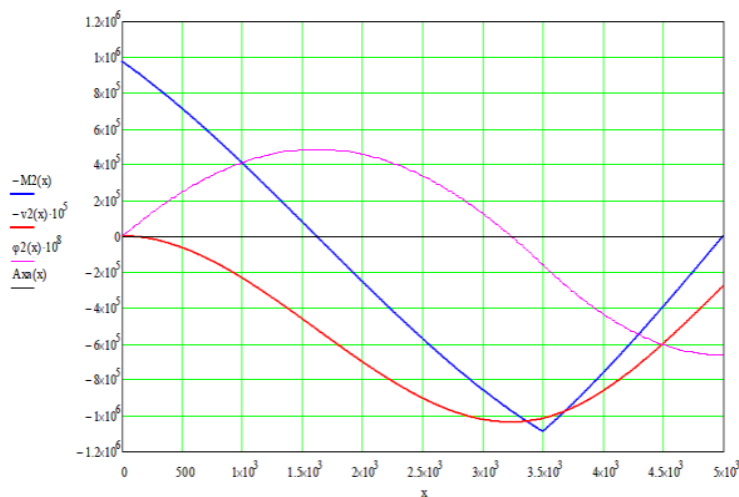

Figure 12: Variation of bending moment, displacements and rotations for $\alpha=0,7 x_{\max }=3234,3$ $\mathrm{mm} ; V_{2 \max }=10,4003 \mathrm{~mm}$.

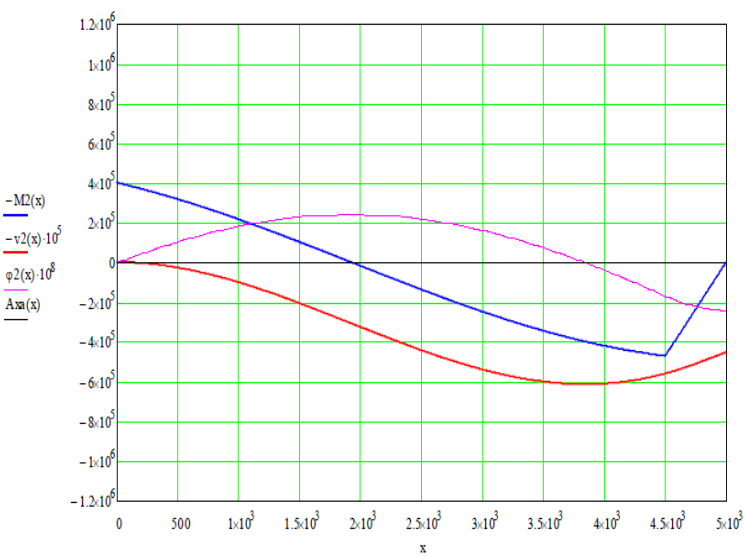

Figure 13: Variation of bending moment, displacements and rotations for $\alpha=0,9 \quad x_{\max }=3845,3$ $m m ; V_{2 \max }=6,1485 \mathrm{~mm}$.

Table 1

\begin{tabular}{|c|c|c|}
\hline \multicolumn{3}{|c|}{ Variation of maximum displacement } \\
\hline$\alpha$ & $v_{\max }$ (First Theory) si $v_{2 \max }$ (Second theory) \\
\hline$\alpha, 1$ & 0,6312 & $v_{2 \max }$ \\
\hline 0,2 & 2,1692 & 0,8165 \\
\hline 0,3 & 4,1102 & 2,8578 \\
\hline 0,4 & 5,9993 & 5,4856 \\
\hline 0,5 & 7,446 & 8,0657 \\
\hline 0,6 & 8,149 & 10,0195 \\
\hline 0,7 & 7,9418 & 10,8839 \\
\hline 0,8 & 6,8942 & 10,4003 \\
\hline 0,9 & 5,3605 & 8,6689 \\
\hline
\end{tabular}

Table 2

\begin{tabular}{|c|c|c|}
\hline \multicolumn{3}{|c|}{$\begin{array}{c}\text { Variation of maximum displacement (frame } \\
0.55 \ldots 0.7 \text { ) } \\
v_{1 \text { max }} \text { (First Theory) si } v_{2 \max } \text { (Second theory) } \\
\alpha=0.55 \ldots \alpha=0.70\end{array}$} \\
\hline$\alpha$ & $V_{1 \max }$ & $V_{2 \max }$ \\
\hline 0,55 & 7,9049 & 10,6105 \\
\hline 0,56 & 7,9716 & 10,6917 \\
\hline 0,57 & 8,0295 & 10,7599 \\
\hline 0,58 & 8,0785 & 10,8148 \\
\hline 0,59 & 8,1183 & 10,8562 \\
\hline 0,6 & 8,149 & 10,8839 \\
\hline 0,61 & 8,1703 & 10,8978 \\
\hline 0,62 & 8,1823 & 10,8976 \\
\hline 0,63 & 8,1847 & 10,8834 \\
\hline 0,64 & 8,1777 & 10,8552 \\
\hline 0,65 & 8,1613 & 10,8131 \\
\hline 0,66 & 8,1355 & 10,7573 \\
\hline 0,67 & 8,1006 & 10,688 \\
\hline 0,68 & 8,0565 & 10,6052 \\
\hline 0,69 & 8,0035 & 10,5093 \\
\hline 0,7 & 7,9418 & 10,4003 \\
\hline
\end{tabular}

\section{Conclusions}

From the numerical results obtained for the particular case presented in this chapter it can be concluded that the method of second-order theory is an accurate compared to those obtained through the first-order theory and the simulation by increasing of $\alpha$ parameter indicate increasing and decreasing of deformations as shown in Figures9 ... 13; this result can allow to find the distance $\alpha L$ of transversal force $Q$ which displacement is the maximum (Table 1 corresponding to Figure 15 and Table 2 corresponding to Figure 14). By simulating the displacement variation for the two theories, the position of force $Q$ was obtained so that the displacement was maxim; the position of section corresponding to maximum displacement is not same for the two theories:

1. The first theory: $\alpha L\left(v_{\operatorname{lmax}}\right)=0,63 \mathrm{~L}$; $v_{\max }=8,1847 \mathrm{~mm}$

2. The second Theory: $\alpha L\left(v_{2 \max }\right)=0,61 \mathrm{~L}$; $v_{2 \max }=10,8978 \mathrm{~mm}$ 
The First and Second-Order Theory of Shearing and Compression in Case of the Beam Fixed at One End and Supported to Helical Spring at the Other End

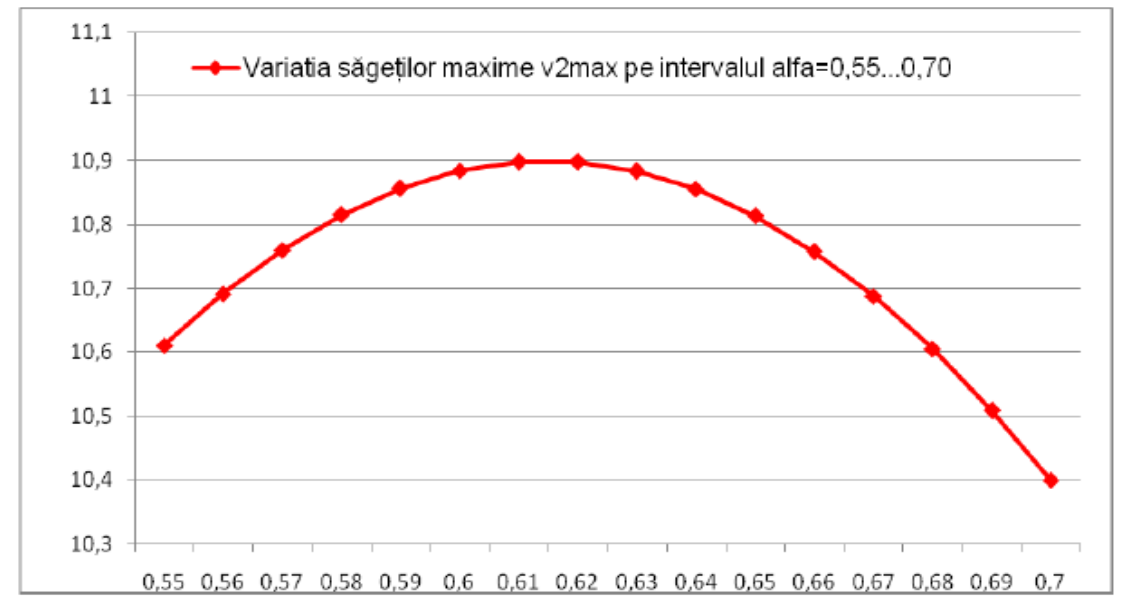

Figure 14: The variation of Maximum displacement v2max depending to $\alpha$

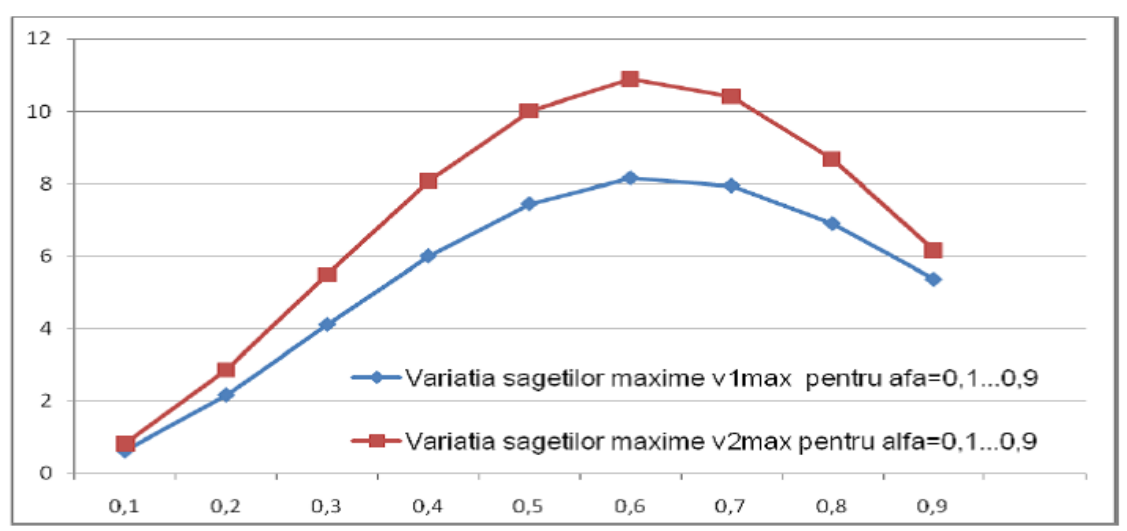

Figure 15: Maximum displacement obtained by first and second theory

\section{References}

[1] Simulescu, I. - Buckling and instability - Lecture 17, Ed. U.T.C.B Bucuresti 2007, ch. 6

[2] Anghel, A. - Rezistenţa materialelor. Partea I . Ed. Universității din Ploiesti, Ploiesti 2004

[3] Anghel, A. - Rezistenţa materialelor. Partea a II-a. Ed. Universității din Ploiesti, 2005
[4] Marin, C. - Aplicaţii ale teoriei elasticităţii şi plasticităţii în inginerie, Ed. Biblioth., 2007.

[5] Marin, C. - Probleme de rezistenţa materialelor rezolvate in MATHCAD. Ed. Bibliotheca Targoviste, 2012

[6] Marin, C. - Rezistenţa materialelor. Partea ISolictări simple, Ed. Bibliotheca, 2013. 